\title{
Reliability of photographs for grading trachoma in field studies
}

\author{
Dana Center \\ for Preventive \\ Ophthalmology of The \\ Wilmer Institute and The \\ School of Public Health, \\ The Johns Hopkins \\ University, Baltimore, \\ MD \\ Sheila K West \\ Hugh R Taylo \\ Correspondence to: Dr Sheila \\ West, Wilmer Room 116 \\ Johns Hopkins Hospital, 600 \\ N Wolfe Street, Baltimore, \\ MD 21205, USA. \\ Accepted for publication \\ 30 June 1989
}

\author{
Sheila K West, Hugh R Taylor
}

\begin{abstract}
The validity and reliability of the use of photographs to assess active trachoma and scarring was studied in a village where the disease was hyperendemic. One hundred and thirty six subjects were graded clinically and had photographs taken of the upper tarsal plate. Agreement between the clinical grade in the field and the grade on the photograph was very good for follicular trachoma, severe trachoma, and scarring ( $x$ statistic values were $0.71,0.74$, and 0.73 respectively). In field studies photographic documentation of trachoma appears to be a valid and reliable approach.
\end{abstract}

Trachoma, a chronic conjunctivitis caused by Chlamydia trachomatis, is a major cause of blindness for much of the developing world. ${ }^{1}$ The assessment clinically of its presence and severity has until recently been difficult because of the lack of a reliable grading scheme. The World Health Organisation published a new, simplified grading scheme ${ }^{2}$ which has been shown to give reproducible trachoma grading among ophthalmologists and ophthalmic nurses after appropriate instruction. ${ }^{3}$ This grading scheme has been used in cross-sectional surveys of risk factors for trachoma ${ }^{4}$ and is being assessed for its utility in longitudinal assessments of change in trachoma over time.

However, relying on the clinical assessment of a longitudinal change in trachoma may be less than optimal for several reasons. First, the clinical examiner may be aware of which subjects have received a specific treatment, and this may subconsciously bias his grading. Secondly, the grader may be aware of the initial grading at the time of follow-up. Thirdly, clinical grading, even if done reliably at each time point, may drift over time in the absence of objective monitoring. For these reasons it might be advantageous to have photographic documentation of a subject's trachoma status at each time; the photographs could then be graded at one time in a masked fashion. However, the reliability of using photographs to detect follicles, scars, and the inflammation of intense disease as defined by the new WHO grading scheme has not been shown.

The purpose of this study was to determine the reliability of a single photograph of the upper tarsus to reproduce the clinical findings as compared with the clinical grade by an experienced trachoma grader.

\section{Methods}

A series of 136 subjects in a trachoma endemic region of East Africa were graded clinically for the presence of follicular trachoma (TF), intense inflammatory trachoma (TI), and trachomatous scarring (TS) in accordance with the WHO new grading scheme. ${ }^{2}$ This grading scheme has been shown to be reproducible when used to grade subjects clinically. In the previous study the $x$ statistic indicating the intraobserver agreement was, on average, 0.74 for TF, 0.77 for TI, and 0.76 for TS.

A photograph of the upper tarsal plate for the right and left eye of each subject was taken with a single lens reflex camera with a macro lens and Kodakchrome ASA 25 film which gave a 1:1 magnification. The film was developed by Kodak Central Laboratories in Rockville, MD. Each subsequent slide was graded by one of us (HRT) who had clinically examined each subject; he was unaware of the original clinical grades assigned to the eye. Photographs were graded by placing them on an illuminated box and observing them with a $2 \cdot 5 \times$ loupe. ${ }^{5}$

The signs of trichiasis (TT) and corneal opacity (CO) were not included in this study because these signs are not likely to change dramatically in most people over a relatively short time. The signs which can fluctuate indicate active trachoma (TF, TI) and scarring (TS).

All subjects gave verbal consent for the clinical examination and photography in accordance with approval by the Joint Committee on Clinical Investigation of the Johns Hopkins Medical Institution. All subjects with active severe trachoma were treated after examination.

The data were entered into an IBM-AT computer and analysed by a $\varkappa$ statistic to assess the percentage agreement, adjusted for agreement due to chance alone. ${ }^{6}$

\section{Results}

Five subjects (4\%) had bilateral photographs (10 eyes) which were ungradable. In three photographs the upper eye lid was not adequately everted. In four photographs the focus was bad or the flash reflex obscured the tarsal plate. In three photographs the examiner's hand shadowed the upper lid. Two of these five subjects had clinical grades of TF in both eyes, and three had scarring only (TS). An additional 13 subjects had one photograph which was ungradable, but the photograph of the companion eye could be graded. A total of 249 eyes were evaluated for agreement.

Agreement between the clinical grade and the photograph grade for follicular trachoma and scarring was very good, $x=0.71$ and 0.73 , respectively (Tables I and II). Agreement was more difficult to assess for severe disease because relatively fewer subjects in this series had severe trachoma (Table III). Thus, the high value for 
TABLE I Reliability of photograph grading for follicular trachoma $(T F)$ in 249 eyes

\begin{tabular}{llr}
\hline & \multicolumn{2}{l}{ Photograph grade } \\
\cline { 2 - 3 } & Present & Absent \\
\hline $\begin{array}{l}\text { Clinical grade: } \\
\begin{array}{c}\text { Present } \\
\text { Absent }\end{array}\end{array}$ & 66 & 22 \\
\hline
\end{tabular}

Percentage agreement $=87 \%$.

$x=0.71(95 \%$ confidence interval $=0.62,0.80)$.

TABLE II Reliability of photograph grading for trachomatous scarring (TC) in 249 eyes

\begin{tabular}{lll}
\hline & \multicolumn{2}{l}{ Photograph grade } \\
\cline { 2 - 3 } & Present & Absent \\
\hline $\begin{array}{l}\text { Clinical grade: } \\
\begin{array}{c}\text { Present } \\
\text { Absent }\end{array}\end{array}$ & 163 & 11 \\
\hline
\end{tabular}

Percentage agreement $=89 \%$.

$x=0.73$ (95\% confidence interval $=0.63,0.82)$.

TABLE III Reliability of photograph grading for trachomatous-intense (TI) in 249 eyes

\begin{tabular}{llc} 
& \multicolumn{2}{l}{ Photograph grade } \\
\cline { 2 - 3 } & Present & Absent \\
\hline $\begin{array}{l}\text { Clinical grade: } \\
\begin{array}{l}\text { Present } \\
\text { Absent }\end{array}\end{array}$ & 11 & 0 \\
\hline
\end{tabular}

Percentage agreement $=\mathbf{9 7} \%$.

$x=0.74(95 \%$ confidence interval $=0.56,0.92)$.

percentage agreement, $97 \%$ reflected the majority of negative findings. However, the $x$ value, $0 \cdot 74$, shows very good agreement, and the $95 \%$ confidence interval includes values which are acceptable.

\section{Discussion}

These data suggest that a single photograph of the upper tarsal plate can reliably reproduce the clinical findings of follicular trachoma and scarring. The agreement between the clinical grade and the grade in the photograph for the same subject was comparable to the agreement obtained if the same subject was graded clinically twice by the same observer.

It is worthwhile to note that the photograph grader identified TI in seven eyes where TI was not observed clinically. Reasons for this finding are not clear. The photograph grader had the time to peruse the photograph and decide if the deep tarsal vessels could be seen, time that a clinical examiner often does not have under field conditions. It is unlikely to be the result of altered magnification in the photograph slides, because these were set to be 1:1 magnification. Eyes in the clinical examination and in the slides were both read with identical magnification, $2 \cdot 5 \times$ loupes.

Photographic data for a total of $4 \%$ (five subjects) in this series were lost because of bilateral ungradable photographs. Thirteen other subjects had photographs which were ungradable, but for one eye only. Data from the clinical grading showed agreement in the trachoma profile between the right and left eyes was $91 \%$. Therefore either eye could represent the trachoma status for these individuals. This small loss (4\%) does need to be factored in planning the size of a trachoma study. In order to minimise loss of data both eyes probably should be photographed for field studies.

In summary, photographic documentation of trachoma appears to be a valid and reproducible technique that may be of value for the assessment of change in trachoma over time.

The study was funded by a grant from the Edna McConnell Clark Foundation.

1 Dawson CR, Jones BR, Tarizzo ML. Guide to Trachomo Control. Geneva: World Health Organisation, 1981

2 Thylefors B, Dawson CR, Jones BR, West SK, Taylor HR. A simple system for the assessment of trachoma and its complication. Bull WHO 1987; 65: 477-83.

3 Tayor HR, West SK, Katala S, Foster A. Trachoma: evaluation 作 Bull WHO 1987; 65: 485-8.

4 West SK, Lynch $M$, Turner V, et al. Water availability and trachoma. Bull WHO in press.

5 trachoma. Bull WHO in press. scopically. Am $\mathcal{F}$ Ophthalmol 1986; 101: 498 .

6 Fleiss JL. Statistical methods for rates and proportions. 2nd ed. Bradley RA, Hunter S, Kendall DG, Watson GS, eds. New York: Wiley, 1981: 1-304. 